\title{
Total syntheses and configuration assignments of JBIR-04 and unantimycin A
}

\author{
Yoshinosuke Usuki, Chie Hamada, Tetsuya Satoh
}

\begin{tabular}{|c|l|}
\hline Citation & Organic \& Biomolecular Chemistry, 15(35); 7346-7351 \\
\hline Issue Date & $2017-08-14$ \\
\hline Type & Journal Article \\
\hline Textversion & Author \\
\hline $\begin{array}{c}\text { Supplementary } \\
\text { information }\end{array}$ & Supplementary information is available at https://doi.org/10.1039/C7OB01732D. \\
\hline Rights & $\begin{array}{l}\text { The following article has been accepted by Organic \& Biomolecular Chemistry. } \\
\text { The final published version is available at https://doi.org/10.1039/C7OB01732D . }\end{array}$ \\
\hline DOI & $10.1039 / C 7 O B 01732 D$ \\
\hline
\end{tabular}

Self-Archiving by Author(s)

Placed on: Osaka City University 


\section{Journal Name}

\section{ARTICLE}

Received 00th January 20xx Accepted 00th January 20xx

DOI: $10.1039 / x 0 x \times 00000 x$

www.rsc.org/

\section{Introduction}

Neoantimycin (1), first isolated in 1967 from Streptomyces orinoci ${ }^{1}$, is a ring-extended member of the antimycin class with a 15membered tetralactone moiety (Figure 1). The recent discovery of prunustatin A (2) as a selective GRP78 molecular chaperone

\section{Total Syntheses and Configuration Assignments of JBIR-04 and Unantimycin}

A

\author{
Yoshinosuke Usuki,* Chie Hamada and Tetsuya Satoh
}

First total syntheses of JBIR-04 and unantimycin A have been achieved. Comparison of our spectroscopic data with those reported for natural samples verified the structure of the natural products; $(2 S, 4 S, 6 S, 7 R, 9 S, 28 S)$ configuration was thus assigned via total synthesis.

downregulator highlights the potential of the neoantimycin subfamily as research probes ${ }^{2,3,4}$. This discovery may lead to the development of new approaches for fighting cancer since GRP78 is a molecular chaperone critical to the unfolded protein response. Moreover, it has emerged as a new therapeutic target for drugresistant cancer cells and cancer stem cells ${ }^{5}$.

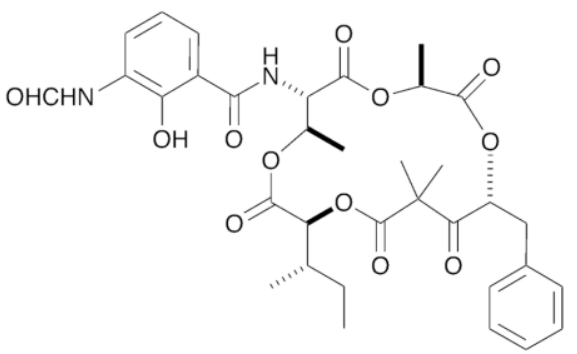

Prunustatin A (2)

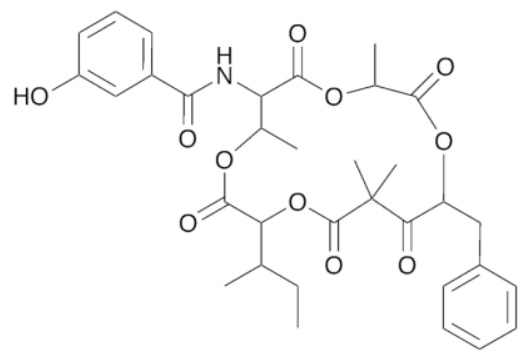

unantimycin $\mathrm{A}(4)$ 
JBIR-04 (3) was isolated from Streptomyces violaceusniger 4541-SVS3 by Shin-ya and coworkers in $2007^{6}$. It showed an inhibitory activity against the GRP78 expression induced by the stimulation of 2-deoxyglucose in HT1080 cells. Analyses of spectroscopic data revealed that the planar structure of $\mathbf{3}$ is a 15membered tetralactone of neoantimycin-class antibiotics. Nevertheless, the absolute stereostructure of $\mathbf{3}$ has not yet been determined. In 2016, Hale reported spectroscopic comparison between natural JBIR-04 and its synthetic compound with a $2 R$ configuration ${ }^{7}$, which is the same as that observed for prunustatin $A$ and SW- $163 A^{8}$, other members of the neoantimycin family. Significant differences were observed between the chemical shifts and coupling constants of $\mathrm{H} 2$ and $\mathrm{H} 12$ : $\mathrm{H} 2$ natural $\delta 5.78$ (dd, $J=$ $10.9,2.9 \mathrm{~Hz}$ ), synthetic $\delta 5.67(\mathrm{t}, J=5.8 \mathrm{~Hz}) ; \mathrm{H} 12$ natural $\delta 3.40(\mathrm{dd}$, $J=14.5,2.9 \mathrm{~Hz}), \delta 3.15(\mathrm{dd}, J=14.5,10.9 \mathrm{~Hz})$, synthetic $\delta 3.35(\mathrm{dd}$, $J=14.0,6.0 \mathrm{~Hz}), \delta 3.26(\mathrm{dd}, J=14.0,5.6 \mathrm{~Hz})$. This result confirms that the absolute stereochemistry of JBIR-04 differs from that of prunustatin $A$; that is, it has a $2 S$ configuration.

Unantimycin A (4), isolated in 2016 by Osada and coworkers from a culture broth of Streptomyces sp. RK88-1355, is a unique member of the neoantimycin family of natural products ${ }^{9}$. The absolute stereostructure of $\mathbf{4}$ has also not yet been determined. The NMR data, including the $\mathrm{H} 12$ chemical shifts and coupling constants, of $\mathbf{4}$ were identical to those of $\mathbf{3}$. The negative values of specific rotation were reported for 3 and $4\left(-28.6^{\circ}\right.$ (c $\left.0.01, \mathrm{MeOH}\right)$ and $-10.8^{\circ}$ ( $\mathrm{c} 0.1, \mathrm{CHCl}_{3}$ ), respectively), indicating that 4 would have the same 15-membered ring as that of $\mathbf{3}$.

As an extension of our synthetic studies on neoantimycin-class antibiotics ${ }^{10}$, we have been engaged in studies directed toward the total synthesis and stereochemical assignment of $\mathbf{3}$ and $\mathbf{4}$. Herein, we report our success in these endeavors.

\section{Results and discussion}

We adopted a synthetic strategy for $\mathbf{3}$ and $\mathbf{4}$, involving cyclization via transesterification of $\beta$-keto ester $\mathbf{7}$ followed by the late-stage introduction of gem-dimethyl groups at C11 (Scheme 1). Metal saltmediated transesterification of $\beta$-keto ester would proceed under mild conditions, which minimizes the epimerization at other stereogenic centers

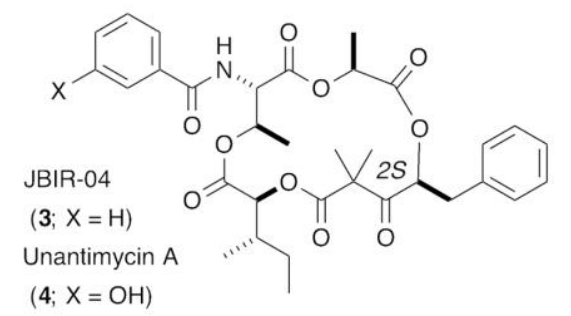<smiles>[X]c1cccc(C(=O)O)c1</smiles>

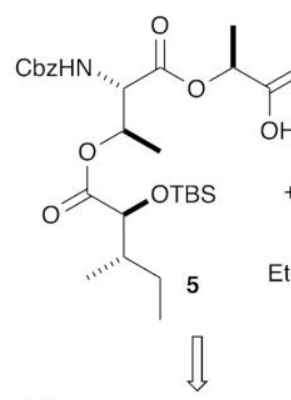<smiles>C=C=CCc1ccccc1CC(O)C(=O)CC(=O)OCC</smiles><smiles>C=CC(=O)N[C@@H](Cc1ccccc1)C(Cc1ccccc1)OC(=O)C(C)OC(=O)[C@@H](C)OC(=O)C(O)[C@H](C)CC</smiles><smiles>CC[C@H](C)C(OC(=O)CC(=O)C(Cc1ccccc1)OC(=O)C(C)OC(=O)[C@@H](NC(=O)OCc1ccccc1)C(C)OC(=O)O)C(=O)O</smiles><smiles>CC[C@H](C)C(N)C(=O)O</smiles><smiles>CC(O)[C@H](N)C(=O)O</smiles><smiles>CC(O)C(=O)O</smiles><smiles>NC(Cc1ccccc1)C(=O)O</smiles>

Our total synthesis of $\mathbf{3}$ and $\mathbf{4}$ commenced with the reaction of aldehyde 10, derived from L-phenylalanine, with ethyl diazoacetate in the presence of $\mathrm{SnCl}_{2}$ (0.13 equiv.) ${ }^{11}$, which provided the corresponding $\beta$-keto ester $\mathbf{1 1}$ in $71 \%$ yield as a ca. 2.6 : 1 mixture of tautomers. Removal of the benzyl ether protecting group using $\mathrm{Pd}(\mathrm{OH})_{2}$ in $\mathrm{EtOH}$ resulted in the formation of 6 in $69 \%$ yield (Scheme 2). 


$$
\text { (2.9 eq) }
$$

Scheme 2 Synthesis of $\beta$-keto ester 6 .

The ring-closure precursor 7 was obtained by condensation of $5^{10 a}$ and 6 in the presence of DCC and DMAP and subsequent removal of the TBS group with $\mathrm{HF}$ in $\mathrm{CH}_{3} \mathrm{CN}$ (Scheme 3). A mixture of $\mathbf{7}$ and anhydrous $\mathrm{CuSO}_{4}$ (20 equiv.) in toluene was heated under reflux. The desired transesterification proceeded smoothly to provide the 15 -membered tetralactone 8 in $73 \%$ yield.
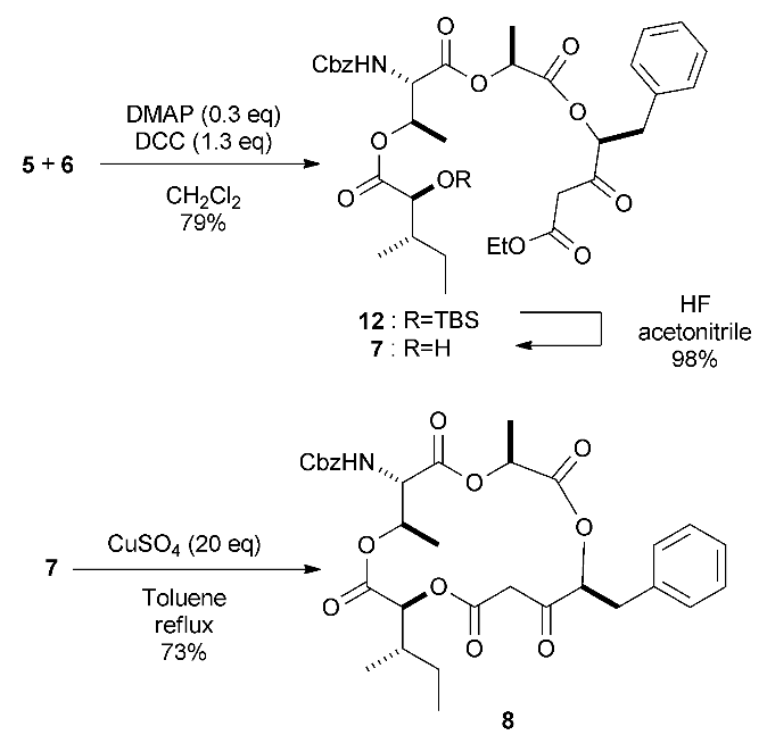

Scheme 3 Preparation and transesterification of $\mathbf{7 .}$

The introduction of gem-dimethyl groups at $\mathrm{C} 11$ was achieved by treatment of 8 with iodomethane (2.9 equiv.) and $\mathrm{Na}_{2} \mathrm{CO}_{3}(9.2$ equiv.) in DMSO at $40^{\circ} \mathrm{C}$ for $4 \mathrm{~h}$ (Scheme 4 ). The desired product 13 was obtained in $54 \%$ yield. To complete the synthesis of $\mathbf{3}$, the $\mathrm{Cbz}$ group of $\mathbf{1 3}$ was removed by hydrogenolysis with $\mathrm{Pd}(\mathrm{OH})_{2}$ in EtOAc to afford $\mathbf{9}$. The subsequent condensation of $\mathbf{9}$ with benzoic acid was achieved using EDCl, HOBt, and NMM in DMF to provide synthetic JBIR-04 (3) in 57\% yield. The spectral data of synthetic 3 were identical to those reported for a natural sample ${ }^{6}$. The optical rotation and melting point of synthetic $3\left([\alpha]_{D}-23.0, \mathrm{c} 0.01, \mathrm{MeOH}\right.$; MP $\left.63^{\circ} \mathrm{C}\right)$ were consistent with those of the natural product $\left([\alpha]_{D}\right.$ $-28.6, \mathrm{c} 0.01, \mathrm{MeOH} ; \mathrm{MP} 60-62^{\circ} \mathrm{C}$ ).
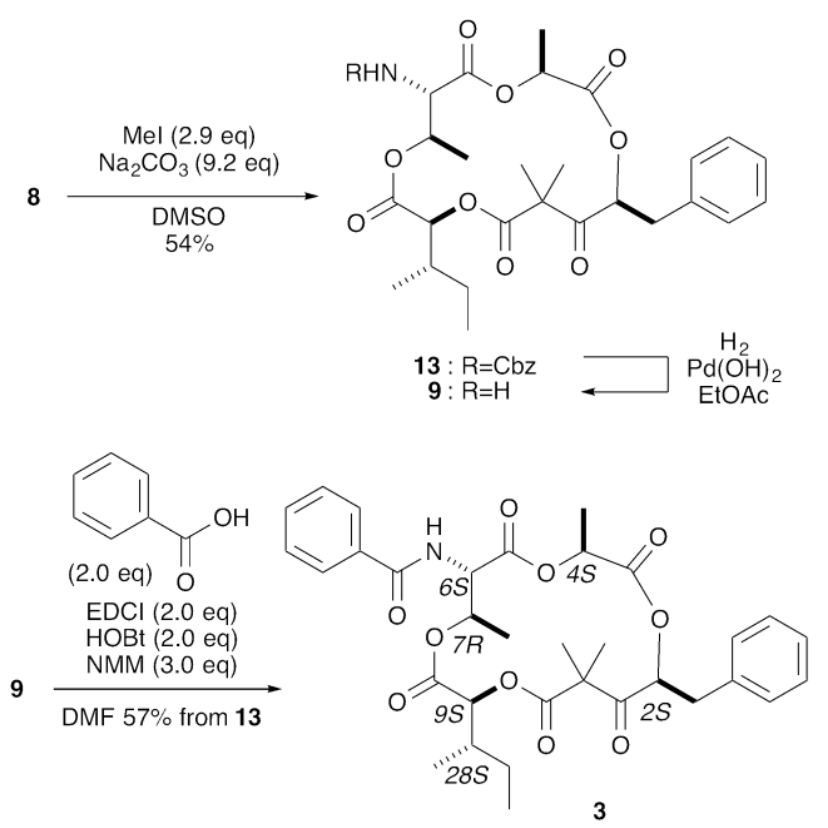

synthetic
$[\alpha]_{\mathrm{D}}-23(\mathrm{c} 0.01, \mathrm{MeOH})$
$\mathrm{MP} 63^{\circ} \mathrm{C}$
natural
$[\alpha]_{\mathrm{D}}-28.6(\mathrm{c} 0.01, \mathrm{MeOH})$
$\mathrm{MP} 60-62^{\circ} \mathrm{C}$
.

Scheme 4 Endgame towards JBIR-04 (3).

We then focused on unantimycin A (4). Condensation of 9 and 3hydroxybenzoic acid was achieved using $\mathrm{EDCl}, \mathrm{HOBt}$, and NMM in DMF to provide synthetic unantimycin A (4) in $55 \%$ yield (Scheme 5). The spectral data of synthetic 4 were identical to those reported for a natural sample ${ }^{9}$. The optical rotation and melting point of synthetic $4\left([\alpha]_{D}-11.9, \mathrm{c} 0.11, \mathrm{CHCl}_{3}\right)$ were consistent with those of the natural product $\left([\alpha]_{\mathrm{D}}-10.8, \mathrm{c} 0.1, \mathrm{CHCl}_{3}\right)$.

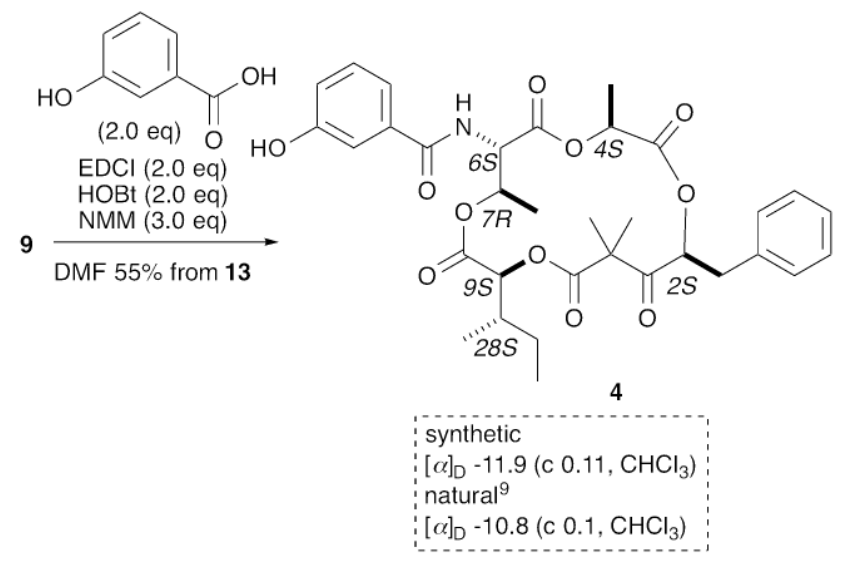

Scheme 5 Endgame towards unantimycin A (4) 


\section{Conclusions}

In summary, the first total syntheses of JBIR-04 and unantimycin A have been achieved. Comparison of our spectroscopic data with those reported for natural samples verified the structure of the natural products; $(2 S, 4 S, 6 S, 7 R, 9 S, 28 S)$ configuration was thus assigned via total synthesis. Further studies on their biological activities are now in progress, and the results will be reported in due course.

\section{Experimental}

\section{General information and materials}

${ }^{1} \mathrm{H}$ and ${ }^{13} \mathrm{C}$ NMR spectra were recorded on either Bruker Avance 300 ( 300 and $75 \mathrm{MHz}$ ), Bruker Avance 400 (400 and $100 \mathrm{MHz}$ ), JEOL ECZ400S (400 and $100 \mathrm{MHz}$ ), or Bruker Avance III 600 (600 and 150 $\mathrm{MHz})$ instruments. Chemical shifts were reported in parts per million (ppm, $\delta$ ) relative to $\mathrm{Me}_{4} \mathrm{Si}(0 \mathrm{ppm})$ and $\mathrm{CDCl}_{3}$ (7.26 ppm for ${ }^{1} \mathrm{H}$ NMR and $77.0 \mathrm{ppm}$ for ${ }^{13} \mathrm{C}$ NMR) as the internal reference. Data are reported as follows: chemical shift, multiplicity $(s=$ singlet, $d=$ doublet, $\mathrm{t}=$ triplet, $\mathrm{q}=$ quartet, $\mathrm{m}=$ multiplet, and $\mathrm{br}=$ broad), coupling constant in $\mathrm{Hz}$, integration. Coupling constants were determined directly from ${ }^{1} \mathrm{H}$ and ${ }^{13} \mathrm{C}$ NMR spectra. Mass spectra were obtained on a JEOL JMS-T100LP (DART, ESI) spectrometer. Infrared (IR) spectra were recorded on a JASCO FT/IR-4600 spectrometer. Optical rotations were measured on a JASCO P-2200 with a path length of $0.1 \mathrm{dm}$ at ambient temperature; the concentrations are reported in $\mathrm{g} / \mathrm{dL}$.

Most of the reagents and solvents were purchased from Wako Pure Chemical Industries, Ltd., Tokyo Kasei Kogyo Co., and SigmaAldrich Co. and were used without further purification unless otherwise noted. All air- and moisture-sensitive reactions were carried out in flame-dried, argon-flushed, two-necked flasks sealed with rubber septa, and dry solvents and reagents were introduced using a syringe. THF was freshly distilled from sodium benzophenone ketyl. Flash column chromatography was carried out on Kanto Chemical silica gel $60 \mathrm{~N}$ (spherical, neutral, 40-50 $\mu \mathrm{m}$ ). Analytical and preparative thin-layer chromatography was performed on Merck precoated silica gel (\#5715 Kieselgel 60F 254 $0.25 \mathrm{~mm}$ and \#5744 Kieselgel $60 \mathrm{~F}_{254} 0.5 \mathrm{~mm}$, respectively).

Ethyl (S)-4-(benzyloxy)-3-oxo-5-phenylpentanoate (11). A solution of aldehyde 10 (1.97 g, $8.24 \mathrm{mmol})$ in $\mathrm{CH}_{2} \mathrm{Cl}_{2}(14 \mathrm{~mL})$ was added to ethyl diazoacetate $(2.68 \mathrm{~g}, 23.5 \mathrm{mmol})$ in $\mathrm{CH}_{2} \mathrm{Cl}_{2}(14 \mathrm{~mL})$ and anhydrous $\mathrm{SnCl}_{2}(209 \mathrm{mg}, 1.10 \mathrm{mmol})$ sequentially at $0^{\circ} \mathrm{C}$. After being stirred overnight at $0^{\circ} \mathrm{C}$, the reaction mixture was washed with aq. sat. $\mathrm{NaHCO}_{3}$ before being dried over $\mathrm{Na}_{2} \mathrm{SO}_{4}$ and concentrated. The residue was purified by flash column chromatography (10\% EtOAc in $n$-hexane) to give $\beta$-keto ester $\mathbf{1 1}$ (ca. $2.6: 1$ mixture of tautomers) as a pale yellow oil (1.92 g, 5.88 mmol, $71 \%$ ): $[\alpha]_{\mathrm{D}}=-50.4$ (c 1.33, $\mathrm{CHCl}_{3}$ ); Major (Keto form): ${ }^{1} \mathrm{H}$ NMR $\left(400 \mathrm{MHz}, \mathrm{CDCl}_{3}\right) \delta 7.34-7.00(10 \mathrm{H}, \mathrm{m}), 4.51-4.34(2 \mathrm{H}, \mathrm{ABq}, J=11.7$ $\mathrm{Hz}, v=38.7 \mathrm{~Hz}), 4.18-4.05(1 \mathrm{H}, \mathrm{m}), 4.12(2 \mathrm{H}, \mathrm{q}, J=7.2 \mathrm{~Hz}), 3.58-$ $3.38(2 \mathrm{H}, \mathrm{ABq}, J=15.9 \mathrm{~Hz}, U=43.2 \mathrm{~Hz}), 3.08(1 \mathrm{H}, \mathrm{dd}, J=14.1,4.2$ $\mathrm{Hz}), 2.95(1 \mathrm{H}, \mathrm{dd}, J=14.1,7.8 \mathrm{~Hz}), 1.22(3 \mathrm{H}, \mathrm{t}, J=7.2 \mathrm{~Hz}) ;{ }^{13} \mathrm{C} \mathrm{NMR}$ $\left(100 \mathrm{MHz}, \mathrm{CDCl}_{3}\right) \delta 205.17,167.29,137.04,136.72,129.63,128.50$, $128.46,127.98,127.83,126.83,85.37,73.04,61.35,45.53,38.22$, 14.11; IR (KBr) $v_{\max } 3423,3029,2982,2929,1750,1719,1685,1654$,
$1638,1560,1542,1509,1491,1457,1315,1225,1095,1030,735$, 698, $478 \mathrm{~cm}^{-1}$; HRDARTMS Calcd. For $\mathrm{C}_{20} \mathrm{H}_{23} \mathrm{O}_{4}$ 327.1596: found $327.1588 \quad[\mathrm{M}+\mathrm{H}]^{+}$. Minor (Enol form, diagnostic peaks only): ${ }^{1 \mathrm{H}} \mathrm{NMR}\left(400 \mathrm{MHz}, \mathrm{CDCl}_{3}\right) \delta 12.10(1 \mathrm{H}, \mathrm{s}), 5$ $.27(1 \mathrm{H}, \mathrm{s}), 4.60(1 \mathrm{H}, \mathrm{d}, J=11.9 \mathrm{~Hz}), 4.30(1 \mathrm{H}, \mathrm{d}, J=11.9 \mathrm{~Hz})$, $4.21(2 \mathrm{H}, \mathrm{q}, J=7.1 \mathrm{~Hz}), 1.30(3 \mathrm{H}, \mathrm{t}, J=7.1 \mathrm{~Hz}) ;{ }^{13} \mathrm{C} \mathrm{NMR}(100 \mathrm{MHz}$, $\left.\mathrm{CDCl}_{3}\right) \delta 176.15,172.23,89.19,79.88,73.01,60.30,40.37,26.91$.

Ethyl (S)-4-hydroxy-3-oxo-5-phenylpentanoate (6). To a solution of $11(509 \mathrm{mg}, 1.56 \mathrm{mmol})$ in ethanol $(20 \mathrm{~mL}), \mathrm{Pd}(\mathrm{OH})_{2}(224$ $\mathrm{mg}$ ) was added. The resulting suspension was stirred overnight under $\mathrm{H}_{2}$. Then, the mixture was filtered through a pad of Celite ${ }^{\circledR}$. The filtrate was concentrated in vacuo. Purification by flash column chromatography (20\% EtOAc in $n$-hexane) afforded 6 as a yellow oil $(254 \mathrm{mg}, 1.08 \mathrm{mmol}, 69 \%):[\alpha]_{\mathrm{D}}=-8.0$ (c $\left.0.87, \mathrm{CHCl}_{3}\right) ;{ }^{1} \mathrm{H} \mathrm{NMR}$ (300 MHz, CDCl 3 ) $\delta 7.35-7.02(5 \mathrm{H}, \mathrm{m}), 4.46(1 \mathrm{H}, \mathrm{dd}, J=8.1,4.5 \mathrm{~Hz})$, $4.17(2 \mathrm{H}, \mathrm{q}, J=7.2 \mathrm{~Hz}), 3.60-3.36(2 \mathrm{H}, \mathrm{m}), 3.14(1 \mathrm{H}, \mathrm{dd}, J=14.1,4.5$ $\mathrm{Hz}), 2.87(1 \mathrm{H}, \mathrm{dd}, J=14.1,8.0 \mathrm{~Hz}), 1.26(3 \mathrm{H}, \mathrm{t}, J=7.2 \mathrm{~Hz}) .{ }^{13} \mathrm{C} \mathrm{NMR}$ $\left(75 \mathrm{MHz}, \mathrm{CDCl}_{3}\right) \delta 204.91,167.11,136.33,129.40,128.69,127.06$, 77.54, 61.69, 45.65, 39.74, 14.08; IR (KBr) $v_{\max } 3449,3032,2983$, $2931,1735,1718,1708,1686,1654,1647,1627,1560,1542,1509$, 1457, 1399, 1368, 1320, 1091, 1029, 942, 730, 701, $477 \mathrm{~cm}^{-1}$; HRDARTMS Calcd. For $\mathrm{C}_{13} \mathrm{H}_{16} \mathrm{NaO}_{4}$ 259.0946: found 259.0961 $[\mathrm{M}+\mathrm{Na}]^{+}$.

Ethyl (S)-4-(( $\left(5^{\prime} S, 8^{\prime} R, 9^{\prime} S, 12^{\prime} S\right)-9^{\prime}-$ (((benzyloxy)carbonyl)amino)-5'-((S)-sec-butyl)-2', 2', 3', 3', , $^{\prime}, 12^{\prime}$ hexamethyl-6',10'-dioxo-4', $7^{\prime}$, ,1' $^{\prime}$-trioxa-3'-silatridecan-13'oyl)oxy)-3-oxo-5-phenylpentanoate (12). To a stirred mixture of 5 (269 mg, $0.49 \mathrm{mmol}), 6$ (128 mg, $0.54 \mathrm{mmol}$ ), and DMAP (19 mg, $0.16 \mathrm{mmol}$ ) in $\mathrm{CH}_{2} \mathrm{Cl}_{2}(2.7 \mathrm{~mL})$, a solution of DCC (188 $\mathrm{mg}, 0.68$ $\mathrm{mmol})$ in $\mathrm{CH}_{2} \mathrm{Cl}_{2}(2.7 \mathrm{~mL})$ was added dropwise at $0^{\circ} \mathrm{C}$. The reaction mixture was stirred at r.t. overnight. The reaction mixture was diluted with $n$-hexane and filtered through a pad of Celite ${ }^{\circledR}$. The filtrate was concentrated in vacuo. The crude residue was purified by flash column chromatography (15\% EtOAc in $n$-hexane) to give 12 as a colorless oil (295 mg, $0.38 \mathrm{mmol}, 79 \%):[\alpha]_{\mathrm{D}}=-18.8$ (c 0.51 , $\mathrm{CHCl} 3) ;{ }^{1} \mathrm{H}$ NMR $\left(400 \mathrm{MHz}, \mathrm{CDCl}_{3}\right) \delta 7.38-7.12(10 \mathrm{H}, \mathrm{m}), 5.47-5.34$ $(3 \mathrm{H}, \mathrm{m}), 5.13(2 \mathrm{H}, \mathrm{s}), 5.07(1 \mathrm{H}, \mathrm{q}, J=7.0 \mathrm{~Hz}), 4.52(1 \mathrm{H}, \mathrm{dd}, J=9.7$, $3.0 \mathrm{~Hz}), 4.13(2 \mathrm{H}, \mathrm{q}, J=7.0 \mathrm{~Hz}), 4.05-3.97(1 \mathrm{H}, \mathrm{m}), 3.55-3.40(2 \mathrm{H}$, $\mathrm{ABq}, J=16.5 \mathrm{~Hz}, v=43.9 \mathrm{~Hz}), 3.36-3.23(1 \mathrm{H}, \mathrm{m}), 3.18(1 \mathrm{H}, \mathrm{dd}, J=$ $14.5,5.2 \mathrm{~Hz}), 3.04(1 \mathrm{H}, \mathrm{dd}, J=14.5,7.3 \mathrm{~Hz}), 1.80-1.67(1 \mathrm{H}, \mathrm{m}), 1.50-$ $1.11(8 \mathrm{H}, \mathrm{m}), 0.92-0.78(6 \mathrm{H}, \mathrm{m}), 0.00(6 \mathrm{H}, \mathrm{s}) ;{ }^{13} \mathrm{C}$ NMR $(100 \mathrm{MHz}$, $\left.\mathrm{CDCl}_{3}\right) \delta 199.30,172.24,169.27,168.93,166.26,156.49,136.07$, $135.03,129.51,128.76,128.65,128.38,128.18,127.36,79.14$, $76.18,70.64,69.51,67.46,61.67,57.77,46.56,39.47,36.55,25.79$, $23.89,18.25,17.12,16.77,15.66,14.14,11.72,-4.87,-5.49$; IR (KBr) $v_{\max } 3448,3067,3033,2960,2935,2857,1752,1734,1685$, $1654,1637,1541,1509,1457.1381,1253,1187,1138,1090,1065$, $1003,876,838,778,738,698,485 \mathrm{~cm}^{-1}$; HRDARTMS Calcd. For $\mathrm{C}_{40} \mathrm{H}_{58} \mathrm{NO}_{12} \mathrm{Si} 772.3728$ : found $772.3728[\mathrm{M}+\mathrm{H}]^{+}$.

Ethyl (S)-4-(((S)-2'-((N-)((benzyloxy)carbonyl)-O-((2'S,3'S)-2'hydroxy-3'-methylpentanoyl)-L-threonyl)oxy)propanoyl)oxy)-3oxo-5-phenylpentanoate (7). To a solution of $12(377 \mathrm{mg}, 0.49$ $\mathrm{mmol})$ in acetonitrile $(8.8 \mathrm{~mL})$ at $0^{\circ} \mathrm{C}, 46.0-48.0 \% \mathrm{HF}$ aq. $(1.3 \mathrm{~mL})$ was added. The reaction mixture was stirred at r.t. for $4 \mathrm{~h}$, before being quenched with sat. $\mathrm{NaHCO} 3$ and extracted with EtOAc. The combined organic layers were washed with brine, dried over 
$\mathrm{Na}_{2} \mathrm{SO}_{4}$, and concentrated in vacuo. The product was used in the next step without further purification (315 $\mathrm{mg}, 0.48 \mathrm{mmol}, 98 \%$ ): $[\alpha]_{\mathrm{D}}=-3.28$ (c $\left.0.53, \mathrm{CHCl}_{3}\right) ;{ }^{1} \mathrm{H} \mathrm{NMR}\left(400 \mathrm{MHz}, \mathrm{CDCl}_{3}\right) \delta 7.42-7.07$ $(10 \mathrm{H}, \mathrm{m}), 5.55(1 \mathrm{H}, \mathrm{dq}, J=6.4,2.8 \mathrm{~Hz}), 5.44-5.34(2 \mathrm{H}, \mathrm{m}), 5.15(2 \mathrm{H}$, s), $5.09(1 \mathrm{H}, \mathrm{q}, J=7.1 \mathrm{~Hz}), 4.58(1 \mathrm{H}, \mathrm{dd}, J=9.7,2.8 \mathrm{~Hz}), 4.16(2 \mathrm{H}, \mathrm{q}$, $J=7.1 \mathrm{~Hz}), 4.00-3.89(1 \mathrm{H}, \mathrm{m}), 3.57-3.43(2 \mathrm{H}, \mathrm{ABq}, J=16.4 \mathrm{~Hz}, \mathrm{U}=$ $38.2 \mathrm{~Hz}), 3.18(1 \mathrm{H}, \mathrm{dd}, J=14.4,5.5 \mathrm{~Hz}), 3.01(1 \mathrm{H}, \mathrm{dd}, J=14.4,7.5$ $\mathrm{Hz}), 2.95-2.74(1 \mathrm{H}, \mathrm{m}), 1.81-1.68(1 \mathrm{H}, \mathrm{m}), 1.52-1.16(8 \mathrm{H}, \mathrm{m}), 0.98-$ $0.84(6 \mathrm{H}, \mathrm{m}) ;{ }^{13} \mathrm{C}$ NMR $\left(100 \mathrm{MHz}, \mathrm{CDCl}_{3}\right) \delta 198.18,173.74,169.15$, $169.01,166.27,156.54,135.93,134.93,129.39,128.62,128.47$, 128.39, 128.18, 127.29, 79.09, 74.37, 71.39, 69.38, 67.52, 61.70, 57.42, 46.63, 38.75, 36.31, 23.91, 16.71, 16.53, 15.34, 14.03, 11.80; $\mathrm{IR}(\mathrm{KBr}) v_{\max } 3448,3066,3033,2965,2936,2876,1750,1734,1719$, 1654, 1637, 1560, 1541, 1509, 1499, 1457, 1384, 1316, 1211, 1132, $1091,1060,737,699,487 \mathrm{~cm}^{-1}$; HRDARTMS Calcd. For $\mathrm{C}_{34} \mathrm{H}_{44} \mathrm{NO}_{12}$ 658.2864: found $658.2858[\mathrm{M}+\mathrm{H}]^{+}$.

Benzyl ((3S,6R,7S,10S,15S)-15-benzyl-10-((S)-sec-butyl)-3,7dimethyl-2,5,9,12,14-pentaoxo-1,4,8,11-

tetraoxacyclopentadecan-6-yl)carbamate (8). A $300 \mathrm{~mL}$ flask containing a mixture of anhydrous $\mathrm{CuSO}_{4}(748 \mathrm{mg}, 4.69 \mathrm{mmol})$ and $7(154 \mathrm{mg}, 246 \mu \mathrm{mol})$ in toluene $(200 \mathrm{~mL})$ was equipped with a small dropping funnel filled with molecular sieves $4 \mathrm{~A}(7.0 \mathrm{~g})$ and a reflux condenser. The mixture was refluxed at $130^{\circ} \mathrm{C}$ for $5 \mathrm{~h}$. After cooling to r.t., it was filtered to remove $\mathrm{CuSO}_{4}$. The filtrate was concentrated and purified by flash column chromatography $(20 \%$ EtOAc in $n$-hexane) to give 8 as a colorless oil $(105 \mathrm{mg}, 172 \mu \mathrm{mol}$, $73 \%) .[\alpha]_{\mathrm{D}}=2.4$ (c $\left.1.04, \mathrm{CHCl}_{3}\right) ;{ }^{1} \mathrm{H} \mathrm{NMR}\left(300 \mathrm{MHz}, \mathrm{CDCl}_{3}\right) \delta 7.41-$ $7.10(10 \mathrm{H}, \mathrm{m}), 5.60(1 \mathrm{H}, \mathrm{dq}, J=6.4,2.3 \mathrm{~Hz}), 5.54(1 \mathrm{H}, \mathrm{d}, J=9.7 \mathrm{~Hz})$, $5.43(1 \mathrm{H}, \mathrm{dd}, J=9.5,5.4 \mathrm{~Hz}), 5.19-5.11(2 \mathrm{H}, \mathrm{m}), 5.04(1 \mathrm{H}, \mathrm{d}, J=9.2$ $\mathrm{Hz}), 4.61(1 \mathrm{H}, \mathrm{dd}, J=9.8,2.3 \mathrm{~Hz}), 3.35-3.18(2 \mathrm{H}, \mathrm{ABq}, J=14.0 \mathrm{~Hz}, v$ $=55.0 \mathrm{~Hz}), 3.25(1 \mathrm{H}, \mathrm{dd}, J=14.0,5.4 \mathrm{~Hz}), 3.12(1 \mathrm{H}, \mathrm{dd}, J=14.0,9.6$ $\mathrm{Hz}), 2.05-1.91(1 \mathrm{H}, \mathrm{m}), 1.55-1.41(1 \mathrm{H}, \mathrm{m}), 1.38(3 \mathrm{H}, \mathrm{d}, J=6.4 \mathrm{~Hz})$, $1.25(3 \mathrm{H}, \mathrm{d}, J=7.1 \mathrm{~Hz}), 1.21-1.00(1 \mathrm{H}, \mathrm{m}), 0.94-0.82(6 \mathrm{H}, \mathrm{m}) ;{ }^{13} \mathrm{C}$ NMR $\left(75 \mathrm{MHz}, \mathrm{CDCl}_{3}\right) \delta 199.37,169.94,168.88,167.86,165.24$, $156.76,135.96,135.45,129.32,128.75,128.60,128.33,128.14$, $127.22,79.35,76.35,71.79,69.76,67.51,57.57,48.23,36.95$, $35.68,24.36,16.57,16.54,14.20,10.53$; IR $(\mathrm{KBr}) v_{\max } 3428,3370$, $3088,3064,3031,2966,2938,2879,1957,1748,1722,1604,1586$, $1515,1455,1382,1346,1310,1282,1192,1132,1088,1061,1028$, 1004, 913, 844, 775, 734, 700, 648, 602, 577, 502, $468 \mathrm{~cm}^{-1}$; HRESIMS Calcd. For $\mathrm{C}_{32} \mathrm{H}_{36} \mathrm{NO}_{11} 610.2288$ : found 610.2274 [M-H]-.

Benzyl ((3S,6S,7R,10S,15S)-15-benzyl-10-((S)-sec-butyl)3,7,13,13-tetramethyl-2,5,9,12,14-pentaoxo-1,4,8,11-

tetraoxacyclopentadecan-6-yl)carbamate (13). To a solution of $\mathrm{Na}_{2} \mathrm{CO}_{3}(92 \mathrm{mg}, 1.35 \mathrm{mmol})$ in DMSO $(1.4 \mathrm{~mL})$ at r.t., a solution of $\beta$-keto ester $8(56 \mathrm{mg}, 91.6 \mu \mathrm{mol})$ in DMSO $(1.3 \mathrm{~mL})$ was added. Then, Mel $(18 \mu \mathrm{L}, 289 \mu \mathrm{mol})$ was added. The reaction mixture was stirred at r.t. for $4 \mathrm{~h}$, diluted with $\mathrm{H}_{2} \mathrm{O}$, and extracted with $n$ hexane/EtOAc $(1: 1,3 x)$. The combined organic layers were washed with brine, dried over $\mathrm{Na}_{2} \mathrm{SO}_{4}$, and concentrated in vacuo. Purification by flash column chromatography $(20 \%$ EtOAc in $n$ hexane) afforded 13 as a colorless oil (31 mg, $54 \mu \mathrm{mol}, 54 \%)$. $[\alpha]_{D}=$ -23.0 (c $\left.0.94, \mathrm{CHCl}_{3}\right) ;{ }^{1} \mathrm{H}$ NMR $\left(400 \mathrm{MHz}, \mathrm{CDCl}_{3}\right) \delta 7.42-7.19(10 \mathrm{H}$, m), $5.76(1 \mathrm{H}, \mathrm{dd}, J=10.7,3.1 \mathrm{~Hz}), 5.68(1 \mathrm{H}, \mathrm{dq}, J=6.4,2.8 \mathrm{~Hz}), 5.57$ $(1 \mathrm{H}, \mathrm{d}, J=9.6 \mathrm{~Hz}), 5.29(1 \mathrm{H}, \mathrm{q}, J=6.8 \mathrm{~Hz}), 5.14(2 \mathrm{H}, \mathrm{d}, J=3.4 \mathrm{~Hz})$, $4.97(1 \mathrm{H}, \mathrm{d}, J=9.3 \mathrm{~Hz}), 4.63(1 \mathrm{H}, \mathrm{dd}, J=9.7,2.8 \mathrm{~Hz}), 3.37(1 \mathrm{H}, \mathrm{dd}, J$ $=14.0,3.1 \mathrm{~Hz}), 3.12(1 \mathrm{H}, \mathrm{dd}, J=14.0,10.7 \mathrm{~Hz}), 2.03-1.92(1 \mathrm{H}, \mathrm{m})$,
$1.55-1.40(1 \mathrm{H}, \mathrm{m}), 1.40(3 \mathrm{H}, \mathrm{s}), 1.38(3 \mathrm{H}, \mathrm{d}, J=6.4 \mathrm{~Hz}), 1.25(3 \mathrm{H}, \mathrm{d}$, $J=6.8 \mathrm{~Hz}), 1.24(3 \mathrm{H}, \mathrm{s}), 1.22-1.06(1 \mathrm{H}, \mathrm{m}), 0.88(3 \mathrm{H}, \mathrm{d}, J=6.9 \mathrm{~Hz})$, $0.86(3 \mathrm{H}, \mathrm{t}, J=7.2 \mathrm{~Hz}) ;{ }^{13} \mathrm{C} \mathrm{NMR}\left(100 \mathrm{MHz}, \mathrm{CDCl}_{3}\right) \delta 203.40,171.21$, $168.87,168.30,168.15,156.78,136.37,135.88,129.41,128.60$, 128.37, 128.33, 128.11, 126.90, 78.77, 75.58, 71.36, 69.58, 67.51, $57.87,53.80,37.40,36.13,24.36,22.35,20.57,16.42,16.19,13.86$, 10.23; IR (KBr) $v_{\max } 3377,3065,3031,2967,2938,2879,1751,1718$, $1654,1637,1604,1541,1509,1499,1456,1383,1342,1314,1281$, $1242,1194,1138,1102,1059,1005,987,912,864,776,734,699$, 647, 549, $487 \mathrm{~cm}^{-1}$; HRESIMS Calcd. For $\mathrm{C}_{34} \mathrm{H}_{40} \mathrm{NO}_{11} 638.2601$ : found $638.2581[\mathrm{M}+\mathrm{H}]^{+}$.

(3S,6S,7R,10S,15S)-6-Amino-15-benzyl-10-((S)-sec-butyl)3,7,13,13-tetramethyl-1,4,8,11-tetraoxacyclopentadecane2,5,9,12,14-pentaone (9). To a solution of benzyl carbamate 13 (17 $\mathrm{mg}, 27 \mu \mathrm{mol})$ in EtOAc $(2 \mathrm{~mL}), 20 \% \mathrm{Pd}(\mathrm{OH})_{2}(6 \mathrm{mg})$ was added. The resulting suspension was stirred overnight under $\mathrm{H}_{2}$. Then, the mixture was filtered through a pad of Celite ${ }^{\circledR}$. The filtrate was concentrated to give amine 9 (13 mg, $26 \mu \mathrm{mol})$. The product was used in the next step without further purification. $[\alpha]_{D}=-90.3$ (c $\left.0.65, \mathrm{CHCl}_{3}\right) ;{ }^{1} \mathrm{H}$ NMR $\left(400 \mathrm{MHz}, \mathrm{CDCl}_{3}\right) \delta 7.32-7.17(5 \mathrm{H}, \mathrm{m}), 5.75$ $(1 \mathrm{H}, \mathrm{dd}, J=10.7,3.1 \mathrm{~Hz}), 5.67(1 \mathrm{H}, \mathrm{dq}, J=6.5,2.7 \mathrm{~Hz}), 5.30(1 \mathrm{H}, \mathrm{q}, J$ $=6.7 \mathrm{~Hz}), 4.99(1 \mathrm{H}, \mathrm{d}, J=9.4 \mathrm{~Hz}), 3.53(1 \mathrm{H}, \mathrm{d}, J=2.7 \mathrm{~Hz}), 3.37(1 \mathrm{H}$, $\mathrm{dd}, J=14.4,3.0 \mathrm{~Hz}), 3.27(1 \mathrm{H}, \mathrm{dd}, J=14.4,10.7 \mathrm{~Hz}), 2.04-1.90(1 \mathrm{H}$, m), 1.53-1.42 (1H, m), $1.45(3 \mathrm{H}, \mathrm{d}, J=6.5 \mathrm{~Hz}), 1.39(3 \mathrm{H}, \mathrm{s}), 1.24-$ $1.06(1 \mathrm{H}, \mathrm{m}), 1.22(3 \mathrm{H}, \mathrm{d}, J=6.8 \mathrm{~Hz}), 1.22(3 \mathrm{H}, \mathrm{s}), 0.88(3 \mathrm{H}, \mathrm{d}, J=6.7$ $\mathrm{Hz}), 0.86(3 \mathrm{H}, \mathrm{t}, J=7.5 \mathrm{~Hz}) ;{ }^{13} \mathrm{C}$ NMR $\left(100 \mathrm{MHz}, \mathrm{CDCl}_{3}\right) \delta 203.76$, $172.12,171.28,169.24,168.66,135.65,129.59,128.43,126.90$, $79.00,75.80,71.63,69.23,58.48,53.81,37.53,36.21,24.49,22.53$, $20.59,16.60,16.51,13.95,10.37$; IR (KBr) $v_{\max } 3221,3064,3030$, $2967,2937,2879,2363,1750,1718,1654,1637,1603,1560,1542$, $1508,1497,1456,1387,1343,1261,1194,1153,1103,1054,1014$, 986, 647, 548, 488, $468 \mathrm{~cm}^{-1}$; HRESIMS Calcd. For $\mathrm{C}_{26} \mathrm{H}_{36} \mathrm{NO}_{9}$ 506.2390: found $506.2437[\mathrm{M}+\mathrm{H}]^{+}$.

JBIR-04 (3). To a solution of 9 (11 mg, $22 \mu \mathrm{mol})$ in DMF (1 mL), benzoic acid ( $12 \mathrm{mg}, 98 \mu \mathrm{mol}), \mathrm{HOBt}(6 \mathrm{mg}, 46 \mu \mathrm{mol}), \mathrm{EDCl} \bullet \mathrm{HCl}(9$ $\mathrm{mg}, 46 \mu \mathrm{mol})$, and NMM ( $8 \mu \mathrm{L}, 73 \mu \mathrm{mol})$ were added successively. The reaction mixture was stirred at r.t. for $14 \mathrm{~h}$, diluted with $\mathrm{H}_{2} \mathrm{O}$, and extracted with EtOAc (3x). The combined organic layers were washed with sat. aq. $\mathrm{NaHCO}_{3}$ and brine, dried over $\mathrm{Na}_{2} \mathrm{SO}_{4}$, and concentrated in vacuo. Purification by flash column chromatography (40\% EtOAc in $n$-hexane) afforded 3 as a colorless amorphous solid $(9.3 \mathrm{mg}, 15 \mu \mathrm{mol}, 57 \%) . \mathrm{MP}=63^{\circ} \mathrm{C}$ (lit. ${ }^{4} \mathrm{MP}=60$ $\left.62^{\circ} \mathrm{C}\right) .[\alpha]_{\mathrm{D}}=-23$ (c $\left.0.01, \mathrm{MeOH}\right)$ (lit. ${ }^{6}[\alpha]_{\mathrm{D}}=-28.6$ (c 0.01, MeOH)). ${ }^{1} \mathrm{H}$ NMR $\left(600 \mathrm{MHz}, \mathrm{CDCl}_{3}\right) \delta 7.91(2 \mathrm{H}, \mathrm{d}, J=7.3 \mathrm{~Hz}), 7.57(1 \mathrm{H}, \mathrm{t}, J=$ $7.4 \mathrm{~Hz}), 7.50(2 \mathrm{H}, \mathrm{t}, J=7.6 \mathrm{~Hz}), 7.31-7.25(5 \mathrm{H}, \mathrm{m}), 6.93(1 \mathrm{H}, \mathrm{d}, J=$ $9.0 \mathrm{~Hz}), 5.79(1 \mathrm{H}, \mathrm{dd}, J=10.7,3.0 \mathrm{~Hz}), 5.78(1 \mathrm{H}, \mathrm{dq}, J=6.5,3.0 \mathrm{~Hz})$, $5.33(1 \mathrm{H}, \mathrm{q}, J=6.8 \mathrm{~Hz}), 5.21(1 \mathrm{H}, \mathrm{dd}, J=9.0,3.0 \mathrm{~Hz}), 5.05(1 \mathrm{H}, \mathrm{d}, J=$ $9.3 \mathrm{~Hz}), 3.40(1 \mathrm{H}, \mathrm{dd}, J=14.5,3.0 \mathrm{~Hz}), 3.15(1 \mathrm{H}, \mathrm{dd}, J=14.5,10.7$ $\mathrm{Hz}), 2.07-1.98(1 \mathrm{H}, \mathrm{m}), 1.56-1.47(1 \mathrm{H}, \mathrm{m}), 1.42(3 \mathrm{H}, \mathrm{s}), 1.41(3 \mathrm{H}, \mathrm{d}$, $J=6.5 \mathrm{~Hz}), 1.26(3 \mathrm{H}, \mathrm{d}, J=6.8 \mathrm{~Hz}), 1.22-1.12(1 \mathrm{H}, \mathrm{m}), 1.25(3 \mathrm{H}, \mathrm{s})$, $0.90(3 \mathrm{H}, \mathrm{d}, J=6.8 \mathrm{~Hz}), 0.89(3 \mathrm{H}, \mathrm{t}, J=7.7 \mathrm{~Hz}) ;{ }^{13} \mathrm{C}$ NMR $(150 \mathrm{MHz}$, $\left.\mathrm{CDCl}_{3}\right) \delta 203.39,171.37,168.84,168.38,168.29,167.97,136.34$, $133.44,132.16,129.42,128.74,128.39,127.27,126.92,78.87$, $75.65,71.82,69.70,55.92,53.76,37.44,36.13,30.90,24.36,22.48$, $20.50,16.41,16.36,13.92,10.24$; IR (KBr) $v_{\max } 3423,3067,3034$, 2967, 2936, 2877, 1752, 1719, 1686, 1655, 1647, 1638, 1579, 1561, $1542,1526,1509,1458,1381,1349,1320,1240,1191,1131,1103$, 
1060, 1009, 971, 887, 700, 624, 540, 505, 482, 462, $438 \mathrm{~cm}^{-1}$; HRESIMS Calcd. For $\mathrm{C}_{33} \mathrm{H}_{39} \mathrm{NNaO}_{10}$ 632.2472: found 632.2472 $[\mathrm{M}+\mathrm{Na}]^{+}$.

Unantimycin A (4). To a solution of $9(7.9 \mathrm{mg}, 16 \mu \mathrm{mol})$ in DMF (0.8 mL), 3-hydroxybenzoic acid $(4.3 \mathrm{mg}, 31 \mu \mathrm{mol}), \mathrm{HOBt}(4.2 \mathrm{mg}$, $31 \mu \mathrm{mol}), \mathrm{EDCl} \bullet \mathrm{HCl}(6.0 \mathrm{mg}, 31 \mu \mathrm{mol})$, and NMM $(5.2 \mu \mathrm{L}, 47 \mu \mathrm{mol})$ were added successively. The reaction mixture was stirred at r.t. for $17 \mathrm{~h}$, diluted with $\mathrm{H}_{2} \mathrm{O}$, and extracted with EtOAc $(3 \mathrm{x})$. The combined organic layers were washed with sat. aq. $\mathrm{NaHCO}_{3}$ and brine, dried over $\mathrm{Na}_{2} \mathrm{SO}_{4}$, and concentrated in vacuo. Purification by flash column chromatography ( $40 \%$ EtOAc in $n$-hexane) afforded 4 as a colorless amorphous solid $(7.0 \mathrm{mg}, 11 \mu \mathrm{mol}, 55 \%)$. MP $=78^{\circ} \mathrm{C}$. $[\alpha]_{\mathrm{D}}=-11.9$ (c $\left.0.11, \mathrm{CHCl}_{3}\right)$, (lit. ${ }^{9}[\alpha]_{\mathrm{D}}=-10.8$ (c 0.1, $\left.\left.\mathrm{CHCl}_{3}\right)\right) .{ }^{1} \mathrm{H} \mathrm{NMR}$ $\left(600 \mathrm{MHz}, \mathrm{CDCl}_{3}\right) \delta 7.49(1 \mathrm{H}, \mathrm{dd}, J=2.5,1.5 \mathrm{~Hz}), 7.43(1 \mathrm{H}, \mathrm{dt}, J=7.8$, $1.3 \mathrm{~Hz}), 7.36(1 \mathrm{H}, \mathrm{t}, J=7.9 \mathrm{~Hz}), 7.31(4 \mathrm{H}, \mathrm{m}), 7.25(1 \mathrm{H}, \mathrm{m}), 7.06(1 \mathrm{H}$, ddd, $J=7.8,2.5,1.0 \mathrm{~Hz}), 6.98(1 \mathrm{H}, \mathrm{d}, J=9.1 \mathrm{~Hz}), 5.96(1 \mathrm{H}, \mathrm{brs}), 5.79$ $(1 \mathrm{H}, \mathrm{dd}, J=10.8,3.2 \mathrm{~Hz}), 5.78(1 \mathrm{H}, \mathrm{dq}, J=6.3,2.7 \mathrm{~Hz}), 5.34(1 \mathrm{H}, \mathrm{q}, J$ $=6.8 \mathrm{~Hz}), 5.19(1 \mathrm{H}, \mathrm{dd}, J=9.1,2.8 \mathrm{~Hz}), 5.04(1 \mathrm{H}, \mathrm{d}, J=9.2 \mathrm{~Hz}), 3.40$ $(1 \mathrm{H}, \mathrm{dd}, J=14.5,3.1 \mathrm{~Hz}), 3.15(1 \mathrm{H}, \mathrm{dd}, J=14.5,10.8 \mathrm{~Hz}), 2.03(1 \mathrm{H}$, m), $1.51(1 \mathrm{H}, \mathrm{m}), 1.42(3 \mathrm{H}, \mathrm{s}), 1.40(3 \mathrm{H}, \mathrm{d}, J=6.4 \mathrm{~Hz}), 1.26(3 \mathrm{H}, \mathrm{d}, J$ $=7.1 \mathrm{~Hz}), 1.26(3 \mathrm{H}, \mathrm{s}), 1.17(1 \mathrm{H}, \mathrm{m}), 0.90(3 \mathrm{H}, \mathrm{d}, \mathrm{J}=6.8 \mathrm{~Hz}), 0.89(3 \mathrm{H}$, $\mathrm{t}, J=7.5 \mathrm{~Hz}) ;{ }^{13} \mathrm{C}$ NMR $\left(150 \mathrm{MHz}, \mathrm{CDCl}_{3}\right) \delta 203.39,171.47,168.85$, $168.32,168.26,167.89,156.31,136.34,134.80,130.10,129.43$, $128.43,126.96,119.47,119.02,114.70,78.93,75.74,71.74,69.75$, 56.03, 53.80, 37.50, 36.13, 24.36, 22.51, 20.53, 16.46, 16.40, 13.93, 10.26; IR (KBr) $v_{\max } 3422,2965,2932,2879,2751,1793,1751,1735$, $1718,1685,1671,1654,1647,1560,1541,1523,1509,1457,1420$, 1387, 1340, 1319, 1194, 1159, 1139, 1103, 1061, 963, 882, 752, $700,669,486,459,423 \mathrm{~cm}^{-1}$; HRESIMS Calcd. For $\mathrm{C}_{33} \mathrm{H}_{39} \mathrm{NNaO}_{11}$ 648.2421: found $648.2403[\mathrm{M}+\mathrm{Na}]^{+}$.

\section{Acknowledgements}

We are grateful to Professor Kazuo Shin-ya of the National Institute of Advanced Industrial Science and Technology for ${ }^{1} \mathrm{H}$ and ${ }^{13} \mathrm{C}$ spectra of naturally isolated JBIR-04. We also thank Dr. Matsumi Doe, Analytical Division, Osaka City University, for NMR measurements.

\section{Notes and references}

(1) G. Cassinelli, A. Grein, P. Orezzi, P. Pennella, A. Sanfilippo, Archiv für Mikrobiologie 1967, 55, 358.

(2) (a) Y. Umeda, S. Chijiwa, K. Furihata, K. Furihata, S. Sakuda, H. Nagasawa, H. Watanabe, K. Shin-ya, J. Antibiot. 2005, 58, 206; (b) Y. Umeda, K. Furihata, S. Sakuda, H. Nagasawa, K. Ishigami, H. Watanabe, M. Izumikawa, M. Takagi, T. Doi, Y. Nakao, K. Shin-ya, Org. Lett. 2007, 9, 4239.

(3) (a) X. Li, R. Zvanych, S. A. Vanner, W. Wang, N. A. Magarvey, Bioorg. Med. Chem. Lett. 2013, 23, 5123; (b) A. A. Salim, K. J. Cho, L. Tan, M. Quezada, E. Lacey, J. F. Hancock, R. J. Capon, Org. Lett. 2014, 16, 5036; (c) S. Yamakoshi, M. Okamoto, H. Sawamoto, Y. Arai, E. Kawanishi, M. Sasaki, K. Takeda, Curr. Org. Synth. 2017, 14, 299.

(4) J. Liu, X. Zhu, S. J. Kim, W. Zhang, Nat. Prod. Rep. 2016, 33, 1146.
(5) (a) B. Luo, A. S. Lee, Oncogene 2013, 32, 805; (b) P. Pyrko, A. H. Schonthal, F. M. Hoffman, T. C. Chen, A. S. Lee, Cancer. Res. 2007, 67, 9809 .

(6) M. Izumikawa, J. Ueda, S. Chijiwa, M. Takagi, K. Shin-ya, J. Antibiot. 2007, 60, 640.

(7) S. Manaviazar, P. Nockemann, K. J. Hale, Org. Lett. 2016, 18, 2902.

(8) K. Takahashi, E. Tsuda, K. Kurokawa, J. Antibiot. 2001, 54, 867.

(9) C. L. Lim, T. Nogawa, A. Okano, Y. Futamura, M. Kawatani, S. Takahashi, D. Ibrahim, H. Osada, J. Antibiot. 2016, 69, 456.

(10) (a) Y. Usuki, H. Ogawa, K.-i. Yoshida, T. Inaoka, H. lio, Asian J. Org. Chem. 2015, 4, 737; (b) H. Ogawa, H. lio, Y. Usuki, Chem. Lett. 2015, 44, 1214.

(11) C. R. Holmquist, E. J. Roskamp, J. Org. Chem. 1989, 54, 3258. 